\title{
Relativistic corrections of one-nucleon current in low-energy three-nucleon photonuclear reactions
}

\author{
A. Deltuva, ${ }^{1}$ A. C. Fonseca, ${ }^{1}$ and P. U. Sauer ${ }^{2}$ \\ ${ }^{1}$ Centro de Física Nuclear da Universidade de Lisboa, P-1649-003 Lisbon, Portugal \\ ${ }^{2}$ Institut für Theoretische Physik, Leibniz Universität Hannover, D-30167 Hannover, Germany
}

(Received 19 November 2009; published 22 December 2009)

\begin{abstract}
Proton-deuteron radiative capture and two- and three-body photodisintegration of ${ }^{3} \mathrm{He}$ at low energy are described using realistic hadronic dynamics and including the Coulomb force. The sensitivity of the observables to the relativistic corrections of the one-nucleon electromagnetic current operator is studied. Significant effects of the relativistic spin-orbit charge are found for the vector-analyzing powers in the proton-deuteron radiative capture and for the beam-target parallel-antiparallel spin asymmetry in the three-body photodisintegration of ${ }^{3} \mathrm{He}$.
\end{abstract}

DOI: $10.1103 /$ PhysRevC.80.064004

PACS number(s): 25.20.-x, 21.45.-v, 24.70.+s, 25.40.Lw

\section{INTRODUCTION}

Electromagnetic (e.m.) reactions in the three-nucleon $(3 N)$ system have been extensively studied in the past with the aim of testing various models of the hadronic two-nucleon $(2 N)$ and $3 N$ interaction and of the nuclear e.m. current [1-6]. In the nucleon-deuteron $(N d)$ radiative capture at higher energies [4], we observed quite a significant effect of the relativistic spin-orbit correction of the one-nucleon $(1 N)$ charge operator. This is consistent with the findings of Refs. [7-9] for the photodisintegration of the deuteron. However, quite surprisingly, we found a sizable effect of the same relativistic spin-orbit correction also for the proton $(p)$ and deuteron $(d)$ vector-analyzing powers in the low-energy $p d$ radiative capture [10]. The aim of this article is to calculate the observables of the low-energy $3 N$ photonuclear reactions. For $p d$ radiative capture, we compare with existing data. For two- and three-body photodisintegration of ${ }^{3} \mathrm{He}$, we make predictions for experiments that are in the process of being performed at the High Intensity Gamma-ray Source (HIGS) facility [11]. We study the sensitivity of observables to relativistic corrections of the $1 \mathrm{~N}$ charge and spatial current.

In Sec. II, we describe the chosen model for the hadronic interaction and e.m. current. Selected results for the $p d$ radiative capture and photodisintegration of ${ }^{3} \mathrm{He}$ are presented in Sec. III. We summarize in Sec. IV.

\section{DYNAMICS}

The hadronic dynamics is based on the realistic two-baryon coupled-channel potential CD Bonn $+\Delta$ [12] that allows for a virtual excitation of a nucleon to a $\Delta$ isobar and thereby yields effective many-nucleon forces [13]. The nonrelativistic part of the e.m. current is taken over from Ref. [4] and has one-baryon and two-baryon pieces. Besides the standard purely nucleonic part, there are additional parts involving the $\Delta$ isobar, which then make effective $2 N$ and $3 N$ contributions to the e.m. current that are consistent with the effective $2 N$ and $3 N$ forces. Since the underlying potential CD Bonn $+\Delta$ is based on the exchange of standard isovector mesons $\pi$ and $\rho$ and isoscalar mesons $\omega$ and $\sigma$, the same meson exchanges are contained in the effective nucleonic forces and currents. The initial and final $3 \mathrm{~N}$ bound and scattering states are described in the isospin formalism by the exact Faddeev and Alt, Grassberger, and Sandhas three-particle equations [14], respectively, that are solved in momentum space using partial wave decomposition [15]. The Coulomb interaction between charged baryons is important in the considered low-energy reactions and is included using the method of screening and renormalization [16-18]. The current is expanded in electric and magnetic multipoles. The magnetic multipoles are calculated from the one- and two-baryon parts of the spatial current. The electric multipoles use the Siegert form of the current without the longwavelength approximation; assuming current conservation, the dominant parts of the one-baryon convection current and the diagonal $\pi$ - and $\rho$-exchange current are taken into account implicitly in the Siegert part of the electric multipoles by the Coulomb multipoles of the charge density; the remaining non-Siegert part of the electric multipoles not accounted for by the charge density is calculated using explicit one- and two-baryon spatial currents. Although the continuity equation is not exactly fulfilled for the nonlocal potential CD Bonn $+\Delta$ or when the relativistic $1 N$ current corrections are included, the employed calculational scheme, based on the Siegert form of the current, effectively corrects the current nonconservation, as argued in Ref. [19], and is therefore reliable for the observables of low-energy photoreactions considered in this article. We also note that the use of the nonrelativistic hadronic dynamics, together with the relativistic current corrections, is somehow inconsistent. However, in the low-energy reactions, it is natural to expect the effect of the relativistic hadronic dynamics to be small; this expectation is consistent with the results of Ref. [20].

To isolate the effects of various relativistic current corrections, we perform several calculations (all using CD Bonn + $\Delta$ as the hadronic potential and including Coulomb) with a different choice of e.m. current:

(i) The nonrelativistic one- and two-baryon e.m. currents are used as in the standard calculations of Ref. [4], except for the parameters of the single nucleon- $\Delta$ transition current, which are taken from Ref. [21]. The corresponding results in the figures are given by the dash-dotted curves.

(ii) The relativistic spin-orbit correction to the $1 \mathrm{~N}$ charge, as given by Eq. (A11a) of Ref. [4], is included in 

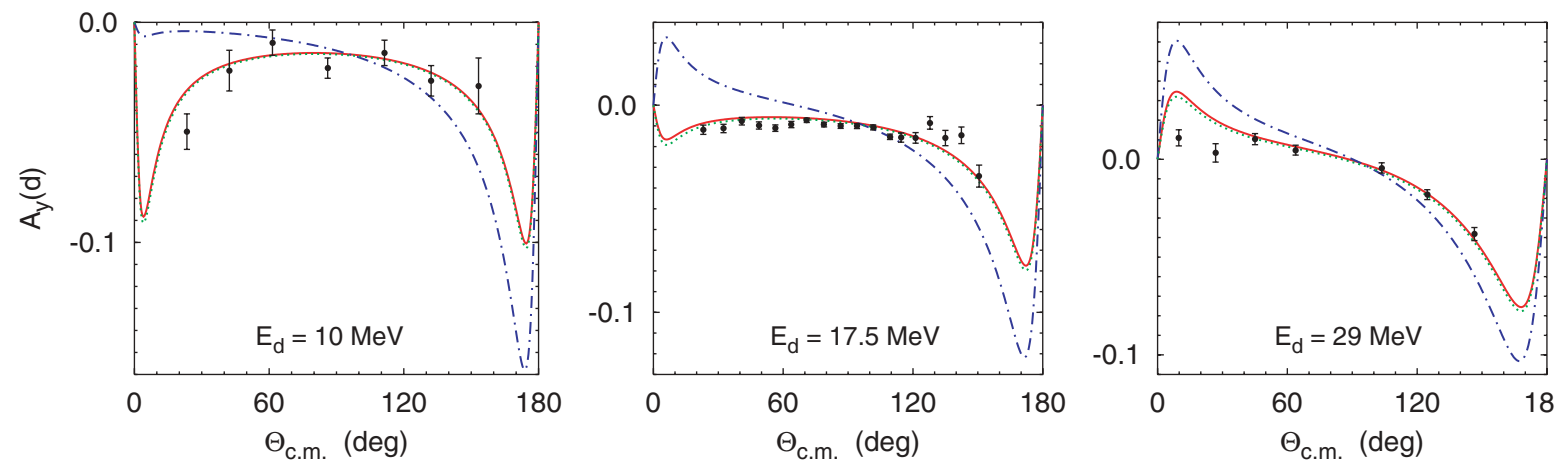

FIG. 1. (Color online) Deuteron vector-analyzing power $A_{y}(d)$ for $p d$ radiative capture at $E_{d}=10,17.5$, and $29 \mathrm{MeV}$ as a function of the c.m. $d-{ }^{3} \mathrm{He}$ scattering angle. Results obtained with nonrelativistic e.m. current (dash-dotted curves), including relativistic spin-orbit charge (solid curves), and including, in addition, relativistic corrections to the spatial current (dotted curves), are compared with the experimental data from Refs. [25-27].

addition to (i); it contributes to the Siegert term. The corresponding results in the figures are given by the solid curves, consistently with Ref. [10].

(iii) With respect to the relativistic Darwin-Foldy correction to the $1 N$ charge, there is a controversy in the literature: according to Ref. [22], it vanishes identically for the reactions with real photons $(\gamma)$, whereas it yields a finite contribution according to Ref. [23]. We include the latter in addition to (ii); however, in all studied lowenergy photoreactions, its effect turns out to be entirely negligible and will therefore not be shown separately.

(iv) In addition to (iii), relativistic corrections to the $1 \mathrm{~N}$ spatial current from Ref. [23] are included; they contribute to the magnetic multipoles and to the non-Siegert part of the electric multipoles. The corresponding results in the figures are given by the dotted curves.

(v) In addition to (iv), the nucleon- $\Delta$ transition charge that is of the relativistic order, as given by Eq. (A11b) of Ref. [4], is included. However, the corresponding results are not shown separately because the effect is entirely negligible.

The technical details of our calculations are explained in Refs. $[4,10,24]$.

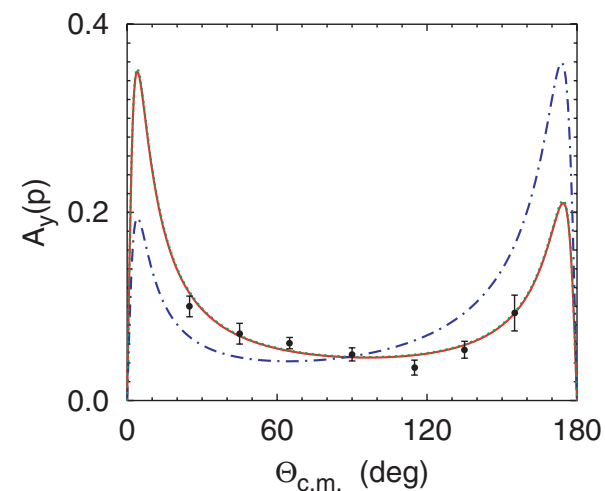

FIG. 2. (Color online) Proton vector-analyzing power $A_{y}(p)$ for $p d$ radiative capture at $E_{p}=5 \mathrm{MeV}$ as a function of the c.m. $p-\gamma$ scattering angle. Curves are as in Fig. 1, and the experimental data are from Ref. [26].

\section{RESULTS}

We start with the $p d$ radiative capture, where significant relativistic effects were predicted in Ref. [10]. In Fig. 1, we show the deuteron vector-analyzing power $A_{y}(d)$ at deuteron laboratory energies $E_{d}=10,17.5$, and $29 \mathrm{MeV}$ as a function of the center-of-mass (c.m.) $d-{ }^{3} \mathrm{He}$ (or $p-\gamma$ ) scattering angle. The effect of the $1 N$ spin-orbit charge is sizable and clearly improves the description of the experimental data, whereas the relativistic corrections of the $1 N$ spatial current contributing to the magnetic multipoles and to the non-Siegert part of the electric multipoles yield only minor changes. Furthermore, it is interesting to note that the spin-orbit charge effect increases with decreasing energy. A strong and beneficial effect is seen also in the proton analyzing power $A_{y}(p)$ at $E_{p}=5 \mathrm{MeV}$ proton laboratory energy in Fig. 2. In contrast, the differential cross section and deuteron tensor analyzing powers at low energies remain almost unaffected by the spin-orbit charge, as can be seen from our previous results [10] at $E_{d}=6 \mathrm{MeV}$; those results are not documented in this article. The spin-orbit charge effect shows up in the deuteron tensor analyzing powers as the energy increases, especially at forward and backward angles, where the differential cross section is small. In Fig. 3, we show $A_{y y}$ at $E_{d}=45 \mathrm{MeV}$;

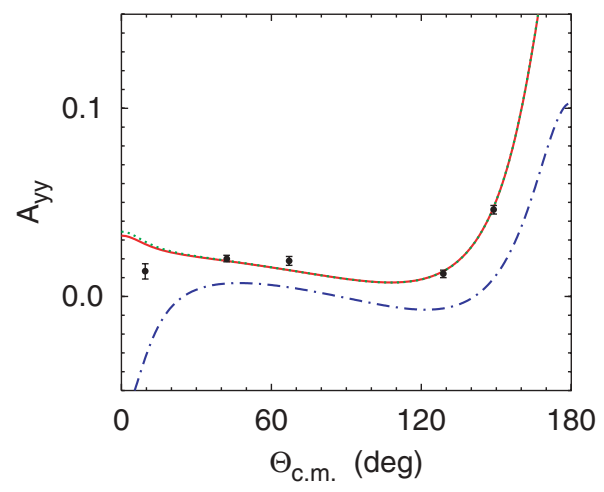

FIG. 3. (Color online) Deuteron tensor analyzing power $A_{y y}$ for $p d$ radiative capture at $E_{d}=45 \mathrm{MeV}$ as a function of the c.m. $d-{ }^{3} \mathrm{He}$ scattering angle. Curves are as in Fig. 1, and the experimental data are from Ref. [27]. 

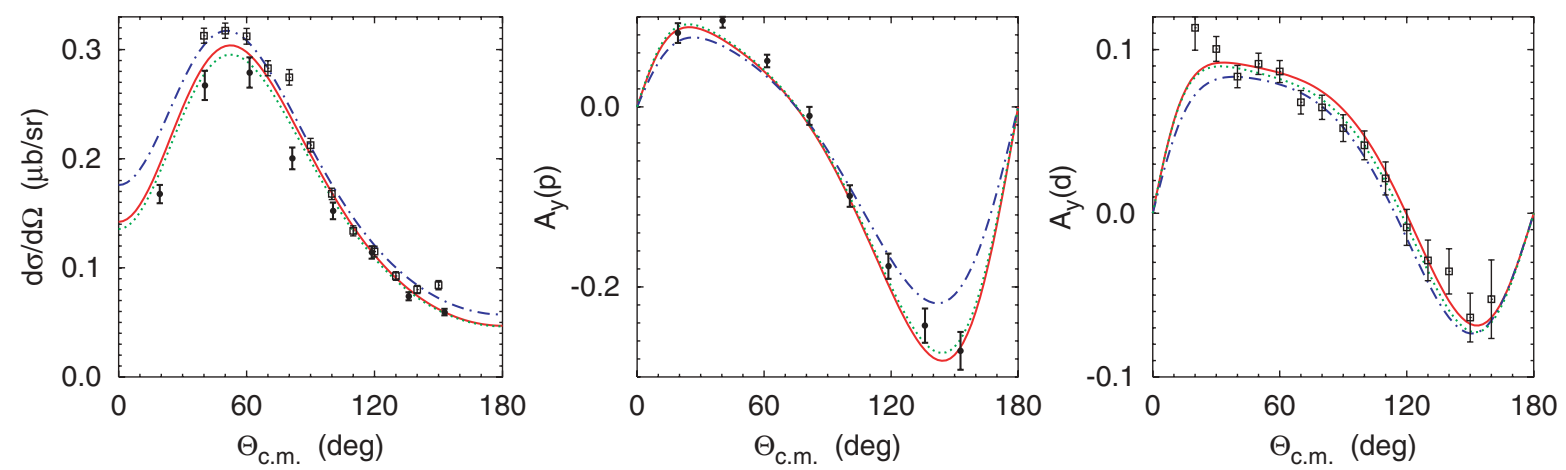

FIG. 4. (Color online) Observables of $p d$ radiative capture at $E_{p}=100 \mathrm{MeV}$ as functions of the c.m. $p$ - $\gamma$ scattering angle. Curves are as in Fig. 1, and the experimental data are from Refs. [30] (circles) and [31] (squares).

further examples can be found in Refs. [10,27]. Finally, we note that our nonrelativistic results, as documented by the dash-dotted curves, are consistent with the results of Ref. [6] derived from the different hadronic interactions and e.m. currents.

One may conjecture that large spin-orbit charge effect in low-energy $A_{y}(p)$ and $A_{y}(d)$ is a result of the high momentum components present in the ${ }^{3} \mathrm{He}$ wave function. We therefore performed additional calculations with AV18 [28] and effective field theory N3LO [29] potentials, which, although having quite comparable ${ }^{3} \mathrm{He}$ binding energies6.923 MeV and 7.128 MeV, respectively-differ significantly in the ${ }^{3} \mathrm{He}$ D-state probability, $8.47 \%$ and $6.32 \%$, and in the expectation value for the ${ }^{3} \mathrm{He}$ internal kinetic energy, $45.68 \mathrm{MeV}$ and $33.78 \mathrm{MeV}$. However, the spin-orbit charge effect turns out to be the same in both cases, thereby ruling out the preceding conjecture. In fact, at low energy, only the matrix elements $\left\langle{ }^{3} \mathrm{He}|E 1| p d\left({ }^{4} P_{J}\right)\right\rangle$ with $J=1 / 2$ and $3 / 2$, that is, the E1 transitions between the ${ }^{4} P_{J} p d$ scattering states and the ${ }^{3} \mathrm{He}$ bound state, calculated using either the Siegert form (as in our standard procedure) or the explicit one- and two-baryon spatial currents, are significantly affected by the relativistic corrections. The nonrelativistic (spin-independent) charge contribution for these matrix elements is suppressed compared to the $\left\langle{ }^{3} \mathrm{He}|E 1| p d\left({ }^{2} P_{J}\right)\right\rangle$ due to the total spin of the ${ }^{3} \mathrm{He}$ bound state being predominantly $\mathcal{S}=1 / 2$. As a consequence, the spin-orbit charge becomes important, especially for the $\left\langle{ }^{3} \mathrm{He}|E 1| p d\left({ }^{4} P_{3 / 2}\right)\right\rangle$, which is responsible for about $90 \%$ of the observed effect in the vector-analyzing powers, which are very sensitive to this particular matrix element.

For curiosity, we show also in Fig. 4 the results at considerably higher energies, that is, $E_{p}=100 \mathrm{MeV}$ or $E_{d}=$ $200 \mathrm{MeV}$, where not only the spin-orbit charge, but also the relativistic corrections of the $1 N$ spatial current, yield visible effects for the differential cross section and spin observables. The description of the experimental data is quite satisfactory, although in this energy regime one can expect further changes due to relativistic hadronic dynamics.

Next we consider the two-body photodisintegration of ${ }^{3} \mathrm{He}$ that is related to the $p d$ radiative capture by time reversal. In Fig. 5, we present results for the linear photon asymmetry $\Sigma$, the target analyzing power $A_{y}\left({ }^{3} \mathrm{He}\right)$, and the beam-target parallel-antiparallel spin asymmetry $A_{\mathrm{P}-\mathrm{A}}$. The importance of the spin-orbit charge is different for these observables: $\Sigma$ remains almost unaffected; $A_{y}\left({ }^{3} \mathrm{He}\right)$ shows moderate effect around the minimum; and $A_{\mathrm{P}-\mathrm{A}}$ is strongly affected, but only at forward and backward angles, where the differential cross section is small. The different effects are due to the different sensitivity of the observables to the crucial matrix elements $\left\langle{ }^{3} \mathrm{He}|E 1| p d\left({ }^{4} P_{J}\right)\right\rangle$, as already encountered in the radiative capture. The effect of the relativistic corrections of the $1 N$ spatial current is negligible for all considered ${ }^{3} \mathrm{He}$ photodisintegration observables and therefore will be not shown for the three-body breakup.
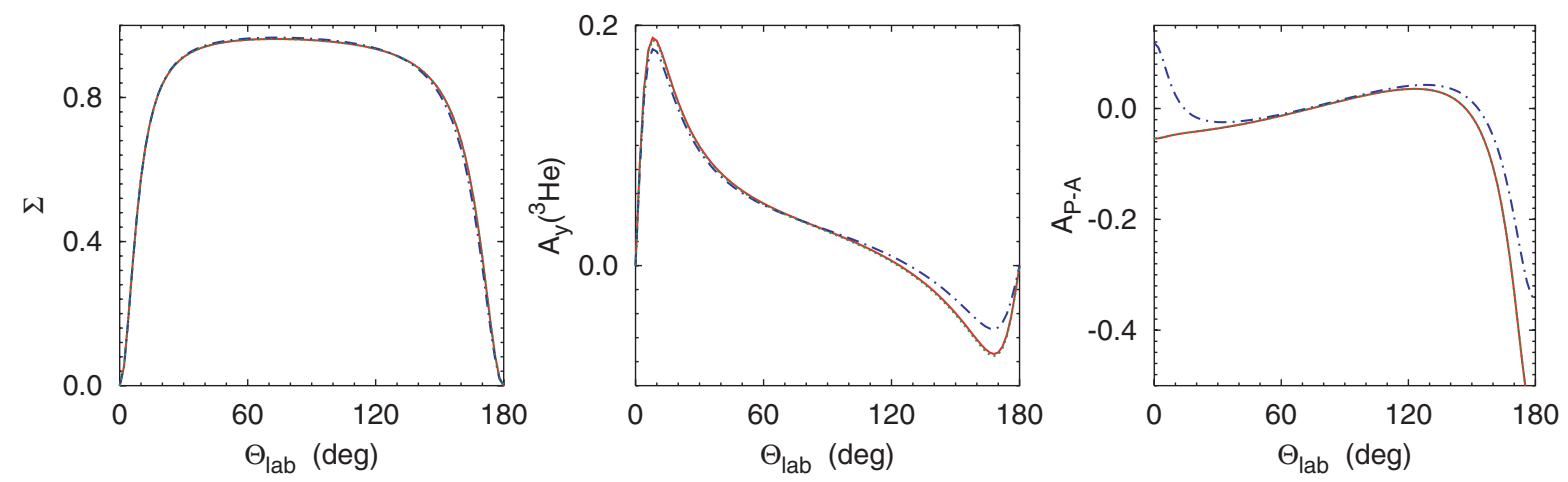

FIG. 5. (Color online) Observables of ${ }^{3} \mathrm{He}$ two-body photodisintegration at $E_{\gamma}=15.2 \mathrm{MeV}$ as functions of the laboratory $p$ - $\gamma$ scattering angle. Curves are as in Fig. 1. 

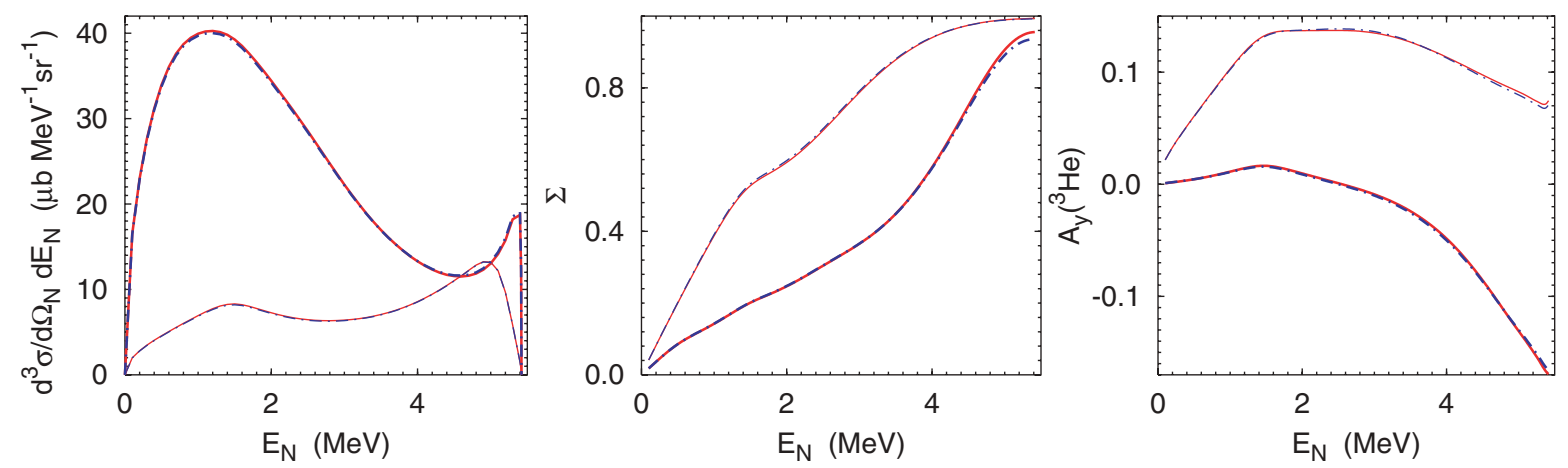

FIG. 6. (Color online) Observables of ${ }^{3} \mathrm{He}$ three-body photodisintegration at $E_{\gamma}=15.2 \mathrm{MeV}$ as functions of the detected nucleon laboratory energy for the nucleon laboratory scattering angle $\Theta_{N}=30^{\circ}$. Results obtained with nonrelativistic e.m. current are given by thick (thin) dash-dotted curves for the detected proton (neutron), whereas the results including relativistic spin-orbit charge are given by thick (thin) solid curves for the detected proton (neutron). Solid and dash-dotted curves lie almost on top of each other.

Finally, in Figs. 6 and 7, we show our predictions for the semi-inclusive observables of the ${ }^{3} \mathrm{He}$ three-body photodisintegration. We note in passing that the inclusion of the Coulomb interaction for this reaction is-among all reactions considered in this article-most important; that fact is also explicitly documented in Refs. [24,32]. The inclusion of the spin-orbit charge yields no visible changes in the semi-inclusive differential cross section and in the spin observables $\Sigma$ and $A_{y}\left({ }^{3} \mathrm{He}\right)$ but leads to a significant effect for the asymmetry $A_{\mathrm{P}-\mathrm{A}}$, which, in contrast to the two-body photodisintegration, is seen at all scattering angles of the detected proton or neutron. Note that the corresponding observable in the deuteron photodisintegration is affected by the relativistic $1 N$ current corrections in a similar way [9].

\section{SUMMARY}

We performed calculations of $3 N$ photonuclear reactions at low energies and studied the effects of various relativistic corrections to the $1 N$ e.m. current operator. The spin-orbit charge is the only important correction when the Siegert form of the electric multipoles is used. The realistic coupled-channel potential CD Bonn $+\Delta$ with Coulomb and the corresponding e.m. current, including relativistic $1 N$ corrections, are quite successful in accounting for the existing $p d$ radiative capture data. Sizable and beneficial effects of the spin-orbit charge were found for proton and deuteron vector-analyzing powers, even at low energies, where the corrections owing to relativistic hadronic dynamics are expected to be small. With increasing energy, the effect of the relativistic $1 N$ current corrections becomes visible for other observables, as well.
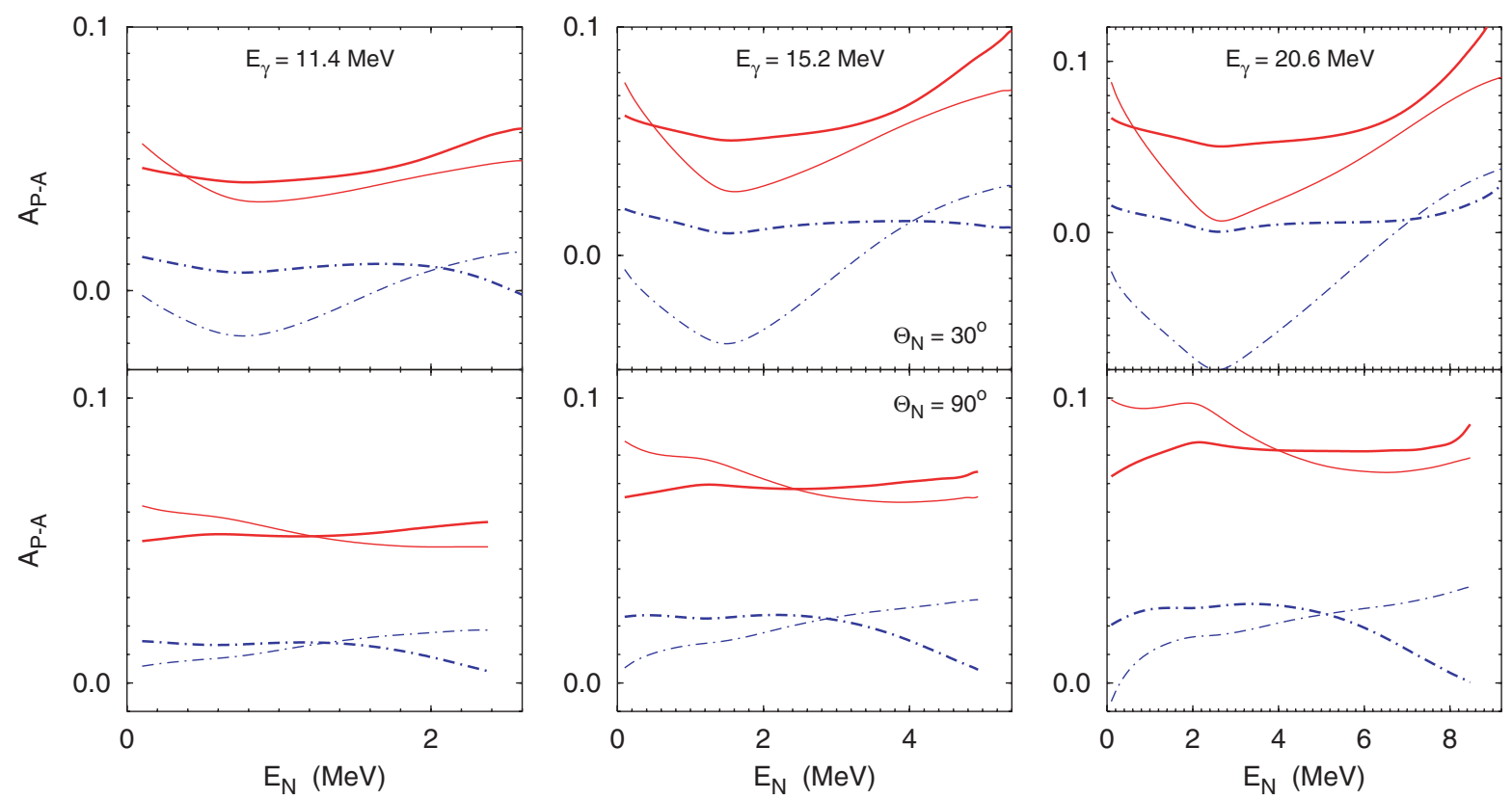

FIG. 7. (Color online) Parallel-antiparallel spin asymmetry of ${ }^{3} \mathrm{He}$ three-body photodisintegration at $E_{\gamma}=11.4,15.2$, and $20.6 \mathrm{MeV}$ as a function of the detected nucleon laboratory energy for (top) $\Theta_{N}=30^{\circ}$ and (bottom) $\Theta_{N}=90^{\circ}$. Curves are as in Fig. 6 . 
Among the observables of the two- and three-body photodisintegration of ${ }^{3} \mathrm{He}$, the beam-target parallel-antiparallel spin asymmetry $A_{\mathrm{P}-\mathrm{A}}$ appears to be most sensitive to the relativistic $1 N$ current corrections. Polarization data do not yet exist for these reactions, but the corresponding experiments are under way at the HIGS facility. The upcoming data will provide crucial tests for the chosen hadronic and e.m. dynamics.
[1] J. Carlson and R. Schiavilla, Rev. Mod. Phys. 70, 743 (1998).

[2] R. Skibiński, J. Golak, H. Kamada, H. Witała, W. Glöckle, and A. Nogga, Phys. Rev. C 67, 054001 (2003).

[3] J. Golak, R. Skibiński, H. Witała, W. Glöckle, A. Nogga, and H. Kamada, Phys. Rep. 415, 89 (2005).

[4] A. Deltuva, L. P. Yuan, J. Adam Jr., A. C. Fonseca, and P. U. Sauer, Phys. Rev. C 69, 034004 (2004).

[5] V. D. Efros, W. Leidemann, G. Orlandini, and E. L. Tomusiak, Phys. Rev. C 69, 044001 (2004).

[6] L. E. Marcucci, M. Viviani, R. Schiavilla, A. Kievsky, and S. Rosati, Phys. Rev. C 72, 014001 (2005).

[7] A. Cambi, B. Mosconi, and P. Ricci, Phys. Rev. Lett. 48, 462 (1982).

[8] F. Ritz, H. Arenhövel, and T. Wilbois, Few-Body Syst. 24, 123 (1998).

[9] H. Arenhövel, Few-Body Syst. 26, 43 (1999).

[10] A. Deltuva, A. C. Fonseca, and P. U. Sauer, Phys. Rev. C 71, 054005 (2005).

[11] H. R. Weller, M. W. Ahmed, H. Gao, W. Tornow, Y. K. Wu, M. Gai, and R. Miskimen, Prog. Part. Nucl. Phys. 62, 257 (2009).

[12] A. Deltuva, R. Machleidt, and P. U. Sauer, Phys. Rev. C 68, 024005 (2003).

[13] A. Deltuva, A. C. Fonseca, and P. U. Sauer, Phys. Lett. B660, 471 (2008).

[14] E. O. Alt, P. Grassberger, and W. Sandhas, Nucl. Phys. B2, 167 (1967).

[15] A. Deltuva, K. Chmielewski, and P. U. Sauer, Phys. Rev. C 67, 034001 (2003).

[16] J. R. Taylor, Nuovo Cimento B 23, 313 (1974); M. D. Semon and J. R. Taylor, Nuovo Cimento A 26, 48 (1975).

[17] E. O. Alt and W. Sandhas, Phys. Rev. C 21, 1733 (1980).
[18] A. Deltuva, A. C. Fonseca, and P. U. Sauer, Annu. Rev. Nucl. Part. Sci. 58, 27 (2008).

[19] A. Deltuva, Ph.D. thesis, University of Hannover, 2003, http:// edok01.tib.uni-hannover.de/edoks/e01dh03/374454701.pdf.

[20] H. Witała, J. Golak, R. Skibiński, W. Glöckle, W. N. Polyzou, and H. Kamada, Phys. Rev. C 77, 034004 (2008)

[21] A. Deltuva, L. P. Yuan, J. Adam Jr., and P. U. Sauer, Phys. Rev. C 70, 034004 (2004).

[22] J. L. Friar, B. F. Gibson, and G. L. Payne, Phys. Rev. C 30, 441 (1984).

[23] F. Ritz, H. Göller, T. Wilbois, and H. Arenhövel, Phys. Rev. C 55, 2214 (1997).

[24] A. Deltuva, A. C. Fonseca, and P. U. Sauer, Phys. Rev. C 72, 054004 (2005).

[25] H. Akiyoshi, K. Sagara, S. Ueno, N. Nishimori, T. Fujita, K. Maeda, H. Nakamura, and T. Nakashima, Phys. Rev. C 64, 034001 (2001).

[26] F. Goeckner, W. K. Pitts, and L. D. Knutson, Phys. Rev. C 45, R2536 (1992).

[27] T. Klechneva et al., Phys. Rev. C 73, 034005 (2006).

[28] R. B. Wiringa, V. G. J. Stoks, and R. Schiavilla, Phys. Rev. C 51, 38 (1995).

[29] D. R. Entem and R. Machleidt, Phys. Rev. C 68, 041001(R) (2003).

[30] M. A. Pickar, H. J. Karwowski, J. D. Brown, J. R. Hall, M. Hugi, R. E. Pollock, V. R. Cupps, M. Fatyga, and A. D. Bacher, Phys. Rev. C 35, 37 (1987).

[31] T. Yagita et al., Mod. Phys. Lett. A 18, 322 (2003).

[32] A. Deltuva, A. C. Fonseca, and P. U. Sauer, Nucl. Phys. A790, $344 c$ (2007). 